\title{
A Batch Arrival Single Server Queue with Server Providing General Service in Two Fluctuating Modes and Reneging during Vacation and Breakdowns
}

\author{
Monita Baruah, ${ }^{1}$ Kailash C. Madan, ${ }^{2}$ and Tillal Eldabi ${ }^{3}$ \\ ${ }^{1}$ Brunel University, UK \\ ${ }^{2}$ College of Information Technology, Ahlia University, Bahrain \\ ${ }^{3}$ Brunel Business School, Brunel University, UK \\ Correspondence should be addressed to Monita Baruah; monitabrh@gmail.com
}

Received 29 July 2013; Revised 4 November 2013; Accepted 13 November 2013; Published 8 January 2014

Academic Editor: Shein-chung Chow

Copyright (C) 2014 Monita Baruah et al. This is an open access article distributed under the Creative Commons Attribution License, which permits unrestricted use, distribution, and reproduction in any medium, provided the original work is properly cited.

We study the behavior of a batch arrival queuing system equipped with a single server providing general arbitrary service to customers with different service rates in two fluctuating modes of service. In addition, the server is subject to random breakdown. As soon as the server faces breakdown, the customer whose service is interrupted comes back to the head of the queue. As soon as repair process of the server is complete, the server immediately starts providing service in mode 1 . Also customers waiting for service may renege (leave the queue) when there is breakdown or when server takes vacation. The system provides service with complete or reduced efficiency due to the fluctuating rates of service. We derive the steady state queue size distribution. Some special cases are discussed and numerical illustration is provided to see the effect and validity of the results.

\section{Introduction}

In this paper, we study a single server queue where the server is providing service in two different modes with probabilities $p_{1}$ and $p_{2}$. In real life, service offered to each arriving unit may not be at the same rate. Sometimes the service offered by the server may be fast, mostly normal, and at times slow. Thus the efficiency of a queuing system is completely affected by different modes of service. In the current age of electronics, most of the servers in a queuing system are electronic devices. It is very natural that such machines may experience sudden mechanical breakdown which cause the stoppage of service until the machine is repaired. In such cases, the customer whose service is interrupted returns back to the head of the queue and waits until repair process is completed. In the real world, we see such breakdown occurring in machines used in production and manufacturing units, communication systems, traffic intersections, automatic teller machines, and so forth.

The server while providing service may take a pause or break which is referred to as vacation in the queuing literature. We have also considered the phenomenon of customers' impatience called reneging. Persons may renege or leave the queue after joining as they become impatient when the server breaks down or during server vacation. Here we assumed reneging to happen during breakdowns and vacation. This is a very realistic situation where we come across situations where customers prefer to leave without receiving service when there is a failure in the service system or the server is not available for a certain period of time.

There have been extensive studies in queues with vacations by prominent researchers since the last decade. Levy and Yechiali [1], Takagi [2], Doshi [3], Madan [4-6], to name a few. We see numerous contributions on queues with breakdown and service interruptions; see, for example, authors like Gaver [7], Aissani and Artalejo [8], Wang et al. [9], Ke [10], Tang [11], and Gray et al. [12]. Recently, Khalaf et al. [13] have studied some queuing systems with random breakdown and delay times. Most of the queues studied here have focused on a single server providing service in the same rate and breakdown occurring during the working state of the server. But in our study, we have assumed that failure or 
breakdown can occur even when the server is in idle state. Also, we further assumed that the server provides service in two fluctuating modes at two different rates.

Further significant contribution has been done on queues dealing with impatient customers like reneging and balking. Queues with reneging had been initially studied by Daley [14]. We find studies on queues with reneging by authors like Altman and Yechiali [15], Bae et al. [16], Ancker and Gafarian [17], Choudhury and Medhi [18], and Zhang et al. [19]. We see real-life implications on reneging during breakdown and vacations as persons waiting for service may discard the queue due to the absence of the server.

The mathematical model is defined under the following assumptions.

(1) Customers (or units) arrive in batches of variable size according to a compound Poisson Process. Let $\lambda c_{k} d t(k=1,2,3, \ldots)$ be the first-order probability of arrival of batch " $k$ " customers in the system at a short interval of time $(t, t+d t]$, where $0 \leq c_{k} \leq 1$, $\sum_{k=1}^{\infty} c_{k}=1 ; \lambda>0$ is the arrival rate of batches.

(2) There is one server providing service in 2 fluctuating modes. The units are served one by one on a first come first served basis. We assume that the probability of providing service in mode 1 is $p_{1}$ and mode 2 is $p_{2}\left(p_{1}+p_{2}=1\right)$. The service time follows general distributions $G_{1}$ and $G_{2}$ at modes 1 and 2 with rates of service $\mu_{1}$ and $\mu_{2}$, respectively. Let $S_{i}(x)$ and $s_{i}(x)$ be the distribution function and density function of the service time, respectively. The conditional probability of the service time during the interval $(x, x+d x)$ given that elapsed service time is $x$ is

$$
\begin{aligned}
& \mu_{i}(x)=\frac{s_{i}(x)}{1-S_{i}(x)} \text { and thus } \\
& s(t)=\mu_{i}(t) \exp \left(-\int_{0}^{t} \mu_{i}(x) d x\right), \quad i=1,2 .
\end{aligned}
$$

(3) The system may fail or be subjected to breakdown at random. The breakdowns are time-homogeneous in the sense that the server can fail even while it is idle. The customer receiving service during breakdown returns back to the head of the queue. Once repair process is complete, the server immediately provides service to the customer in mode $p_{1}$. We assume that time between breakdowns occurs according to a Poisson process with mean rate of breakdown as $\alpha>0$. Further, the repair times follow a general (arbitrary) distribution with distribution function $B(x)$ and density function $b(x)$. Let the conditional probability of completion of the repair process be $\beta(x) d x$ such that $\beta(x)=B(x) /(1-B(x))$ and thus $b(u)=\beta(u) \exp \left(-\int_{0}^{u} \beta(x) d x\right)$.

(4) After each service completion, the server may take a vacation of random length with probability $\phi$ or remain in the system with probability $1-\phi$. The server's vacation time follows a general (arbitrary) distribution with distribution function $F(v)$ and density function $f(v)$. Let $\eta(x) d x$ be the conditional probability of completion of vacation during the interval $(x, x+d x]$ during the elapsed vacation time $x$, so that

$$
\begin{aligned}
& \eta(x)=\frac{f(v)}{1-F(v)} \text { and therefore } \\
& f(v)=\eta(v) \exp \left(-\int_{0}^{v} \eta(x) d x\right) .
\end{aligned}
$$

(5) We assume that customers may renege (leave the system after joining the queue) when the server is on vacation and reneging is assumed to follow exponential distribution with parameter $\gamma$. Thus $f(t)=$ $\gamma e^{-\gamma t} d t, \gamma>0$. Let $\gamma d t$ be the probability that a customer can renege during a short interval of time $(t, t+d t]$

\section{Definitions and Notations}

Let $P_{n}^{(i)}(t, x)=$ Probability that at time $t$ the server is providing service in mode $i,(i=1,2)$ since elapsed time $x$, and there are $n(n \geq 0)$ customers in the queue excluding one customer in service.

$P_{n}^{(i)}(x)=\int_{0}^{\infty} P_{n}^{(i)}(t, x) d x=$ Probability that at time $t$ there are $n(n \geq 0)$ customers in the queue excluding one customer in service irrespective of the value $x(i=1,2)$.

$R_{n}(t, x)=$ Probability that the server is under repairs since elapsed time $x$ and there are $n(n \geq 0)$ customers in the queue.

$R_{n}(t)=\int_{0}^{\infty} R_{n}(t, x) d x=$ Probability that the server is under repairs and there are $n(n \geq 0)$ customers in the queue irrespective of the value $x$.

$V_{n}(t, x)=\int_{0}^{\infty} V(t, x) d x=$ Probability that at time $t$ the server is on vacation with elapsed vacation time $x$ and there are $n(n \geq 0)$ customers waiting in the queue for service.

$V_{n}(t)=\int_{0}^{\infty} V_{n}(t, x) d x=$ Probability that at time $t$ the server is on vacation and there are $n(n \geq 0)$ customers in the queue irrespective of the value $x$.

$Q=$ the steady state probability that the server is idle.

\section{Steady State Equations Governing the System}

According to the assumptions above, we derive the following steady state differential equations:

$$
\begin{gathered}
\frac{d}{d x} P_{n}^{(1)}(x)+\left(\lambda+\mu_{1}(x)+\alpha\right) P_{n}^{(1)}(x) \\
=\lambda \sum_{k=1}^{n} c_{k} P_{n-k}^{(1)}(x) \quad n \geq 1 \\
\frac{d}{d x} P_{0}^{(1)}(x)+\left(\lambda+\mu_{1}(x)+\alpha\right) P_{0}^{(1)}(x)=0
\end{gathered}
$$




$$
\begin{gathered}
\frac{d}{d x} P_{n}^{(2)}(x)+\left(\lambda+\mu_{2}(x)+\alpha\right) P_{n}^{(2)}(x) \\
=\lambda \sum_{k=1}^{n} c_{k} P_{n-k}^{(2)}(x) \quad n \geq 1 \\
\frac{d}{d x} P_{0}^{(2)}(x)+\left(\lambda+\mu_{2}(x)+\alpha\right) P_{0}^{(2)}(x)=0 \\
\frac{d}{d x} R_{n}(x)+(\lambda+\beta(x)+\gamma) R_{n}(x) \\
=\lambda \sum_{k=1}^{n} c_{k} R_{n-k}(x)+\gamma R_{n+1}(x) \quad n \geq 1 \\
\frac{d}{d x} R_{0}(x)+(\lambda+\beta(x)+\gamma) R_{0}(x)=\gamma R_{1}(x) \\
\frac{d}{d x} V_{n}(x)+(\lambda+\eta(x)+\gamma) V_{n}(x) \\
\quad+\int_{0}^{n} \sum_{i=1}^{\infty} c_{n-i} V_{n-i}(x)+\gamma V_{n+1}(x) \quad n \geq 1 \\
\frac{d}{d x} V_{0}(x)+(\lambda+\eta(x)) V_{0}(x)=\gamma V_{0}(x)(x) \mu_{1}(x) d x \\
Q=(1-\phi) \\
\quad(x) d x+\int_{0}^{\infty} V_{0}(x) \eta(x) d x \\
\left.\quad(2)(x) \mu_{2}(x) d x\right]
\end{gathered}
$$

The boundary conditions for solving the above differential equations are

$$
\begin{aligned}
P_{n}^{(1)}(0)= & \lambda c_{n+1} p_{1} Q+(1-\phi) \\
& \times\left[p_{1} \int_{0}^{\infty} P_{n+1}^{(1)}(x) \mu_{1}(x) d x\right. \\
& \left.+p_{1} \int_{0}^{\infty} P_{n+1}^{(2)}(x) \mu_{2}(x) d x\right] \\
& +p_{1} \int_{0}^{\infty} R_{n+1}(x) \beta(x) d x \quad n \geq 0 . \\
& +p_{1} \int_{0}^{\infty} V_{n+1}(x) \eta(x) d x \quad n \geq
\end{aligned}
$$

The left side indicates the probability that there is one customer in service and $n$ customers in the queue. Zero in the parentheses of the left side of the boundary condition (12) implies the moment when the service starts in mode 1. The right side of (12) shows five mutually exclusive cases each contributing to the immediate start of service in mode 1 . The first term on the right side means that as soon as a batch of size $n+1$ arrives when the system is empty, the service in mode 1 starts immediately. Similarly, other terms on the right side of (12) indicate that just after the completion of a service in mode 1 or in mode 2 , or completion of a vacation or completion of repairs, the service in mode 1 immediately starts. Utilizing similar reasoning, we get the other boundary conditions as follows:

$$
\begin{gathered}
P_{n}^{(2)}(0)=\lambda c_{n+1} p_{2} Q+(1-\phi) \\
\quad \times\left[p_{2} \int_{0}^{\infty} P_{n+1}^{(1)}(x) \mu_{1}(x)\right. \\
\left.\quad+p_{2} \int_{0}^{\infty} P_{n+1}^{(2)}(x) \mu_{2}(x) d x\right] \\
+p_{2} \int_{0}^{\infty} R_{n+1}(x) \beta(x) d x \\
+p_{2} \int_{0}^{\infty} V_{n+1}(x) \eta(x) d x \quad n \geq 0 \\
R_{n+1}(0)=\alpha \int_{0}^{\infty} P_{n}^{(1)}(x) d x+\alpha \int_{0}^{\infty} P_{n}^{(2)}(x) d x \\
=\alpha P_{n}^{(1)}+\alpha P_{n}^{(2)} n \geq 0 \\
R_{0}(0)=\alpha Q \\
\quad+\int_{0}^{\infty} P_{n}^{(1)}(x) \mu_{1}(x) d x \\
\left.V_{n}(0)=\phi(x) \mu_{2}(x) d x\right] .
\end{gathered}
$$

\section{Queue Size Distribution at Random Epoch}

Let us now define the following probability generating functions:

$$
\begin{aligned}
& P^{(i)}(x, z)=\sum_{n=0}^{\infty} z^{n} P_{n}^{(i)}(x) \quad P(z)=\sum_{n=0}^{\infty} z^{n} P_{n}^{(i)} \\
& R(x, z)=\sum_{n=0}^{\infty} z^{n} R_{n}(x) \quad R(z)=\sum_{n=0}^{\infty} z^{n} R_{n} \\
& V(x, z)=\sum_{n=0}^{\infty} z^{n} V_{n}(x) \quad V(z)=\sum_{n=0}^{\infty} z^{n} V_{n} \\
& C(z)=\sum_{n=1}^{\infty} z^{n} c_{n}
\end{aligned}
$$

Now multiplying (3) by $z^{n}$ and taking sum over " $n$ " from 1 to $\infty$, adding with (4) and using (17), we obtain

$$
\frac{d}{d x} P^{(1)}(x, z)+\left(\lambda-\lambda C(z)+\mu_{1}(x)+\alpha\right) P^{(1)} \quad(x, z)=0 .
$$


Similarly performing the same operations to (5), (7), and (9) and using (17), we have

$$
\begin{array}{ll}
\frac{d}{d x} P^{(2)}(x, z)+\left(\lambda-\lambda C(z)+\mu_{2}(x)+\alpha\right) P^{(2)} & (x, z)=0 \\
\frac{d}{d x} R(x, z)+\left(\lambda-\lambda C(z)+\beta(x)+\gamma-\frac{\gamma}{z}\right) R & (x, z)=0 \\
\frac{d}{d x} V(x, z)+\left(\lambda-\lambda C(z)+\eta(x)+\gamma-\frac{\gamma}{z}\right) V & (x, z)=0 .
\end{array}
$$

Now for the boundary conditions, we multiply (12) by $z^{n+1}$, summing over " $n$ " from 0 to $\infty$, and using the probability generating functions defined in (17) and (11), we get

$$
\begin{aligned}
z P^{(1)}(0, z)= & p_{1} \lambda C(z) Q+(1-\phi) \\
& \times\left[p_{1} \int_{0}^{\infty} P^{(1)}(x, z) \mu_{1}(x) d x\right. \\
& \left.\quad+p_{1} \int_{0}^{\infty} P^{(2)}(x, z) \mu_{2}(x) d x\right] \\
& +p_{1} \int_{0}^{\infty} R(x, z) \beta(x) d x \\
& +p_{1} \int_{0}^{\infty} V(x, z) \eta(x) d x \\
& -p_{1}\left\{( 1 - \phi ) \left[\int_{0}^{\infty} P_{0}^{(1)}(x) \mu_{1}(x) d x\right.\right. \\
& +\int_{0}^{\infty} R_{0}(x) \beta(x) d x \\
\left.+\int_{0}^{\infty} V_{0}(x) \eta(x) d x\right\} & \left.+\int_{0}^{\infty} P_{0}^{(2)}(x) \mu_{2}(x) d x\right]
\end{aligned}
$$$$
z P^{(1)}(0, z)=p_{1}(\lambda C(z)-\lambda-\alpha) Q+(1-\phi) p_{1}
$$$$
\times\left[\int_{0}^{\infty} P^{(1)}(x, z) \mu_{1}(x) d x\right.
$$$$
\left.+\int_{0}^{\infty} P^{(2)}(x, z) \mu_{2}(x) d x\right]
$$$$
+p_{1} \int_{0}^{\infty} R(x, z) \beta(x) d x
$$$$
+p_{1} \int_{0}^{\infty} V(x, z) \eta(x) d x \text {. }
$$

Similarly, performing the same operation in (13), we have

$$
\begin{aligned}
z P^{(2)}(0, z)= & p_{2}(\lambda C(z)-\lambda-\alpha) Q+(1-\phi) p_{2} \\
& \times\left[\int_{0}^{\infty} P^{(1)}(x, z) \mu_{1}(x) d x\right. \\
& \left.\quad+\int_{0}^{\infty} P^{(2)}(x, z) \mu_{2}(x) d x\right] \\
& +p_{2} \int_{0}^{\infty} R(x, z) \beta(x) d x \\
& +p_{2} \int_{0}^{\infty} V(x, z) \eta(x) d x .
\end{aligned}
$$

Now multiplying (14) by $z^{n+1}$, summing over $n$ from 0 to $\infty$, and using (15) and (17), we have

$$
\begin{aligned}
& R(0, z)-\alpha Q=\alpha z P^{(1)}(z)+\alpha z P^{(2)}(z) \\
& \text { Or } \quad R(0, z)=\alpha z\left[P^{(1)}(z)+P^{(2)}(z)\right]+\alpha Q .
\end{aligned}
$$

Again multiplying by $z^{n}$, summing over $n$ from 0 to $\infty$, in (16) and (17), we get

$$
\begin{aligned}
V(0, z)=\phi\left[\int_{0}^{\infty} P^{(1)}(x, z) \mu_{1}(x) d x\right. \\
\left.+\int_{0}^{\infty} P^{2}(x, z) \mu_{2}(x) d x\right] .
\end{aligned}
$$

Now integrating (17)-(21) over 0 to $x$ gives

$$
\begin{aligned}
P^{(1)}(x, z)= & P^{(1)}(0, z) \\
& \times \exp \left[-(\lambda-\lambda C(z)+\alpha) x-\int_{0}^{x} \mu_{1}(t) d t\right] \\
P^{(2)}(x, z)= & P^{(2)}(0, z) \\
& \times \exp \left[-(\lambda-\lambda C(z)+\alpha) x-\int_{0}^{x} \mu_{2}(t) d t\right]
\end{aligned}
$$

$$
\begin{aligned}
R(x, z)= & R(0, z) \\
& \times \exp \left[-\left(\lambda-\lambda C(z)+\gamma-\frac{\gamma}{z}\right)-\int_{0}^{x} \beta(t) d t\right]
\end{aligned}
$$

$$
\begin{aligned}
V(x, z)= & V(0, z) \\
& \times \exp \left[-\left(\lambda-\lambda C(z)+\gamma-\frac{\gamma}{z}\right)-\int_{0}^{x} \eta(t) d t\right] .
\end{aligned}
$$


Again integrating (26)-(29) by parts with respect to $x$ yields

$$
\begin{gathered}
P^{(1)}(z)=P^{(1)}(0, z)\left[\frac{1-S_{1}^{*}(\lambda-\lambda C(z)+\alpha)}{(\lambda-\lambda C(z)+\alpha)}\right] \\
P^{(2)}(z)=P^{(2)}(0, z)\left[\frac{1-S_{2}^{*}(\lambda-\lambda C(z)+\alpha)}{(\lambda-\lambda C(z)+\alpha)}\right] \\
R(z)=R(0, z)\left[\frac{1-B^{*}(\lambda-\lambda C(z)+\gamma-\gamma / z)}{\lambda-\lambda C(z)+\gamma-\gamma / z}\right] \\
V(z)=V(0, z)\left[\frac{1-F^{*}(\lambda-\lambda C(z)+\gamma-\gamma / z)}{\lambda-\lambda C(z)+\gamma-\gamma / z}\right],
\end{gathered}
$$

where $S_{j}^{*}(\lambda-\lambda C(z)+\alpha)=\int_{0}^{\infty} e^{-(\lambda-\lambda C(z)+\alpha) x} d S_{j}(x), B^{*}(\lambda-$ $C(z)+\gamma-\gamma / z)=\int_{0}^{\infty} e^{-(\lambda-\lambda C(z)+\gamma-\gamma / z) x} d B(x)$ and $F^{*}(\lambda-$ $\lambda C(z)+\gamma-\gamma / z)=\int_{0}^{\infty} e^{-(\lambda-\lambda C(z)+\gamma-\gamma / z) x} d F(x)$ are the LaplaceStieltjes transform of the service time $S_{j}(x), j=1,2$, repair time $B(x)$, and vacation time $F(x)$, respectively.

To evaluate the integrals $\int_{0}^{\infty} P^{(1)}(x, z) \mu_{1}(x) d x, \int_{0}^{\infty} P^{(2)}$ $(x, z) \mu_{2}(x) d x, \int_{0}^{\infty} R(x, z) \beta(x) d x$ and $\int_{0}^{\infty} V(x, z) \eta(x) d x$, we multiply (26), (27), (28), and (29) by $\mu_{1}(x), \mu_{2}(x), \beta(x)$, and $\eta(x)$, respectively, and integrating w.r.t. $x$, we have

$$
\begin{aligned}
& \int_{0}^{\infty} P^{(1)}(x, z) \mu_{1}(x) d x=P^{(1)}(0, z) S_{1}^{*}(\lambda-\lambda C(z)+\alpha) \\
& \int_{0}^{\infty} P^{(2)}(x, z) \mu_{2}(x) d x=P^{(2)}(0, z) S_{2}^{*}(\lambda-\lambda C(z)+\alpha) \\
& \int_{0}^{\infty} R(x, z) \beta(x) d x=R(0, z) B^{*}\left(\lambda-\lambda C(z)+\gamma-\frac{\gamma}{z}\right) \\
& \int_{0}^{\infty} V(x, z) \eta(x) d x=V(0, z) F^{*}\left(\lambda-\lambda C(z)+\gamma-\frac{\gamma}{z}\right) .
\end{aligned}
$$

Let us take $\lambda-\lambda C(z)+\alpha=m, \lambda-\lambda C(z)+\gamma-\gamma / z=k$ and utilizing the values from (34) in (22) and (23), we obtain

$$
\begin{aligned}
z P^{(1)}(0, z)= & -p_{1} m Q+(1-\phi) p_{1} \\
& \times\left\{P^{(1)}(0, z) S_{1}^{*}(m)+P^{(2)}(0, z) S_{2}^{*}(m)\right\} \\
& +p_{1} R(0, z) B^{*}(k)+p_{1} V(0, z) F^{*}(k)
\end{aligned}
$$

Rewrite the above equation as

$$
\begin{aligned}
& {\left[z-(1-\phi) p_{1} S_{1}^{*}(m)\right] P^{(1)}(0, z)} \\
& \quad-(1-\phi) p_{1} S_{2}^{*}(m) P^{(2)}(0, z) \\
& \quad-p_{1} B^{*}(k) R(0, z)-p_{1} F^{*}(k) V(0, z) \\
& =-p_{1} m Q .
\end{aligned}
$$

Similarly, from (23), we get

$$
\begin{aligned}
& -(1-\phi) p_{2} S_{1}^{*}(m) P^{(1)}(0, z) \\
& +\left[z-(1-\phi) p_{2} S_{2}^{*}(m)\right] P^{(2)}(0, z) \\
& -p_{2} B^{*}(k) R(0, z)-p_{2} F^{*}(k) V(0, z)=-p_{2} m Q .
\end{aligned}
$$

Again using relations (30) and (31) in (24) yields

$$
\begin{aligned}
& \alpha z\left[\frac{1-S_{1}^{*}(m)}{m}\right] P^{(1)}(0, z) \\
& \quad+\alpha z\left[\frac{1-S_{2}^{*}(m)}{m}\right] P^{(2)}(0, z)-R(0, z)=-\alpha Q .
\end{aligned}
$$

\section{Utilizing (34) in (25) gives}

$V(0, z)=\phi P^{(1)}(0, z) S_{1}^{*}(m)+\phi P^{(2)}(0, z) S_{2}^{*}(m)$.

Now using (39) in (36) and (37) and rewriting the equations again, we obtain

$$
\begin{aligned}
& {\left[z-p_{1} S_{1}^{*}(m)\left\{(1-\phi)+\phi F^{*}(k)\right\}\right] P^{(1)}(0, z)} \\
& \quad-p_{1} S_{2}^{*}(m)\left\{(1-\phi)+\phi F^{*}(k)\right\} P^{(2)}(0, z) \\
& \quad-p_{1} B^{*}(k) R(0, z)=-p_{1} m Q \\
& \quad-\left\{(1-\phi)+\phi F^{*}(k)\right\} p_{2} S_{1}^{*}(m) P^{(1)}(0, z) \\
& \quad+\left[z-p_{2} S_{2}^{*}(m)\left\{(1-\phi)+\phi F^{*}(k)\right\}\right] P^{(2)}(0, z) \\
& \quad-p_{2} B^{*}(k) R(0, z)=-p_{2} m Q \\
& \frac{\alpha z\left[1-S_{1}^{*}(m)\right]}{m} P^{(1)}(0, z)+\frac{\alpha z\left[1-S_{2}^{*}(m)\right]}{m} P^{(2)}(0, z) \\
& -R(0, z)=-\alpha Q .
\end{aligned}
$$

Solving (40) using Cramer's rule, we obtain

$$
\begin{aligned}
& P^{(1)}(0, z)=\frac{z p_{1}\left[m-\alpha B^{*}(k)\right] Q}{\Delta} \\
& P^{(2)}(0, z)=\frac{z p_{2}\left[m-\alpha B^{*}(k)\right] Q}{\Delta}
\end{aligned}
$$

$R(0, z)$

$$
=\frac{z \alpha Q\left\{p_{1} S_{1}^{*}(m)+p_{2} S_{2}^{*}(m)\right\}\left[\left\{(1-\phi)+\phi F^{*}(k)\right\}-z\right]}{\Delta} .
$$

Thus using (41) and (42) in (39) yields

$$
\begin{aligned}
& V(0, z)=\frac{z \phi Q\left[m-\alpha B^{*}(k)\right]\left[p_{1} S_{1}^{*}(m)+p_{2} S_{2}^{*}(m)\right]}{\Delta}, \\
& \Delta=z^{2}-z\left\{(1-\phi)+\phi F^{*}(k)\right\}\left\{p_{1} S_{1}^{*}(m)+p_{2} S_{2}^{*}(m)\right\} \\
& -z^{2} \alpha B^{*}(k)\left[\frac{p_{1}\left\{1-S_{1}^{*}(m)\right\}}{m}+\frac{p_{2}\left\{1-S_{2}^{*}(m)\right\}}{m}\right] .
\end{aligned}
$$


We now substitute the values from (41)-(44) in (30)-(33) and obtain

$$
\begin{aligned}
& P^{(1)}(z)=\frac{z p_{1}\left[m-\alpha B^{*}(k)\right] Q}{\Delta}\left[\frac{1-S_{1}^{*}(m)}{m}\right] \\
& P^{(2)}(z)=\frac{z p_{2}\left[m-\alpha B^{*}(k)\right] Q}{\Delta}\left[\frac{1-S_{2}^{*}(m)}{m}\right]
\end{aligned}
$$

$R(z)$

$$
\begin{gathered}
=\frac{z \alpha Q\left\{p_{1} S_{1}^{*}(m)+p_{2} S_{2}^{*}(m)\right\}\left[\left\{(1-\phi)+\phi F^{*}(k)\right\}-z\right]}{\Delta} \\
\times\left[\frac{1-B^{*}(k)}{k}\right] \\
V(z)=\frac{z \phi Q\left[m-\alpha B^{*}(k)\right]\left[p_{1} S_{1}^{*}(m)+p_{2} S_{2}^{*}(m)\right]}{\Delta} \\
\quad \times\left[\frac{1-F^{*}(k)}{k}\right] .
\end{gathered}
$$

Let $P_{q}(z)$ denote the probability generating function of the queue size irrespective of the state of the system. Thus adding the equations in (46), we have

$$
P_{q}(z)=P^{(1)}(z)+P^{(2)}(z)+R(z)+V(z)=\frac{N(z)}{D(z)} .
$$

The unknown probability $Q$ can be obtained using the normalizing condition

$$
P_{q}(1)+Q=1
$$

Since $P_{q}(z)$ is of indeterminate of $0 / 0$ form for $z=1$, we apply L'Hopital's rule on (47) and derive

$$
\begin{aligned}
P^{(1)}(1)= & \lim _{z \rightarrow 1} P^{(1)}(z) \\
= & Q p_{1}[\lambda E(I)+\alpha(\lambda E(I)-\gamma) E(R)] \\
& \left.\times\left[\frac{1-S_{1}^{*}(\alpha)}{\alpha}\right]\right) \\
& \times\left(\left[p_{1} S_{1}^{*}(\alpha)+p_{2} S_{2}^{*}(\alpha)\right]\right. \\
& \times\{1-\phi(\lambda E(I)-\gamma) E(V)\} \\
& -[\alpha(\lambda E(I)-\gamma) E(R)+\lambda E(I)] \\
& \left.\times\left\{\frac{p_{1}\left(1-S_{1}^{*}(\alpha)\right)}{\alpha}+\frac{p_{2}\left(1-S_{2}^{*}(\alpha)\right)}{\alpha}\right\}\right)^{-1},
\end{aligned}
$$

$$
\begin{aligned}
& P^{(2)}(1)=\lim _{z \rightarrow 1} P^{(2)}(z) \\
&=( Q p_{2}[\lambda E(I)+\alpha(\lambda E(I)-\gamma) E(R)] \\
&\left.\times\left[\frac{1-S_{2}^{*}(\alpha)}{\alpha}\right]\right) \\
& \times {\left[p_{1} S_{1}^{*}(\alpha)+p_{2} S_{2}^{*}(\alpha)\right] } \\
& \times\{1-\phi(\lambda E(I)-\gamma) E(V)\} \\
&-[\alpha(\lambda E(I)-\gamma) E(R)+\lambda E(I)] \\
&\left.\times\left\{\frac{p_{1}\left(1-S_{1}^{*}(\alpha)\right)}{\alpha}+\frac{p_{2}\left(1-S_{2}^{*}(\alpha)\right)}{\alpha}\right\}\right)^{-1},
\end{aligned}
$$

$$
\begin{aligned}
R(1)=\lim _{z \rightarrow 1} R(z) & \\
\times(\alpha & Q\left\{p_{1} S_{1}^{*}(\alpha)+p_{2} S_{2}^{*}(\alpha)\right\} \\
& \times\{1-(\lambda E(I)-\gamma) \phi E(V)\} E(R)) \\
\times( & {\left[p_{1} S_{1}^{*}(\alpha)+p_{2} S_{2}^{*}(\alpha)\right] } \\
& \times\{1-\phi(\lambda E(I)-\gamma) E(V)\} \\
& -[\alpha(\lambda E(I)-\gamma) E(R)+\lambda E(I)] \\
& \left.\times\left\{\frac{p_{1}\left(1-S_{1}^{*}(\alpha)\right)}{\alpha}+\frac{p_{2}\left(1-S_{2}^{*}(\alpha)\right)}{\alpha}\right\}\right)^{-1}
\end{aligned}
$$

$V(1)$

$$
\begin{aligned}
=(Q \phi & \{\lambda E(I)+\alpha(\lambda E(I)-\gamma) E(R)\} \\
& \left.\times\left\{p_{1} S_{1}^{*}(\alpha)+p_{2} S_{2}^{*}(\alpha)\right\} E(V)\right) \\
\times( & {\left[p_{1} S_{1}^{*}(\alpha)+p_{2} S_{2}^{*}(\alpha)\right] } \\
& \times\{1-\phi(\lambda E(I)-\gamma) E(V)\} \\
& -[\alpha(\lambda E(I)-\gamma) E(R)+\lambda E(I)] \\
& \left.\times\left\{\frac{p_{1}\left(1-S_{1}^{*}(\alpha)\right)}{\alpha}+\frac{p_{2}\left(1-S_{2}^{*}(\alpha)\right)}{\alpha}\right\}\right)^{-1},
\end{aligned}
$$

where $E(I)$ is the mean batch of arrivals, $E(R)$ is the mean repair time, and $E(V)$ is the mean vacation time. 
Now utilize (49), (50), (51), and (52) in the normalizing condition and obtain $Q$ as

$$
\begin{aligned}
Q=( & {\left[p_{1} S_{1}^{*}(\alpha)+p_{2} S_{2}^{*}(\alpha)\right]\{1-\phi(\lambda E(I)-\gamma) E(V)\} } \\
& -[\alpha(\lambda E(I)-\gamma) E(R)+\lambda E(I)] \\
& \left.\times\left[\frac{p_{1}\left(1-S_{1}^{*}(\alpha)\right)}{\alpha}+\frac{p_{2}\left(1-S_{2}^{*}(\alpha)\right)}{\alpha}\right]\right) \\
& \times\left(\{1+\phi \gamma E(V)+\alpha E(R)\}\left\{p_{1} S_{1}^{*}(\alpha)+p_{2} S_{2}^{*}(\alpha)\right\}\right)^{-1} .
\end{aligned}
$$

Thus the utilization factor is obtained as

$$
\begin{aligned}
\rho=1-Q=( & {[\alpha(\lambda E(I)-\gamma) E(R)+\lambda E(I)] } \\
& \times\left[\frac{p_{1}\left(1-S_{1}^{*}(\alpha)\right)}{\alpha}+\frac{p_{2}\left(1-S_{2}^{*}(\alpha)\right)}{\alpha}\right] \\
& +\{\phi \lambda E(I) E(V)-\alpha E(R)\} \\
& \left.\times\left\{p_{1} S_{1}^{*}(\alpha)+p_{2} S_{2}^{*}(\alpha)\right\}\right) \\
\times & ((1+\phi \gamma E(V)+\alpha E(R)) \\
& \left.\times\left\{p_{1} S_{1}^{*}(\alpha)+p_{2} S_{2}^{*}(\alpha)\right\}\right)^{-1} .
\end{aligned}
$$

Equation (53) is the stability condition under which the steady state exists given by $\rho<1$.

We can substitute the value of $Q$ in (49)-(52) and obtain clear and explicit expression for $P^{(i)}(1) ; i=1,2$, the steady state probability that the server is active and providing service to customers in two fluctuating modes, 1 and 2 at any random point of time, $R(1)$ the steady state probability that the server is in failed state and is under repairs, and $V(1)$ the steady state probability that the server goes for vacation at any random point of time.

The probability generating function of the queue length irrespective of whether the server is idle but available in the system or the server is under repairs or whether the server is on vacation is obtained as

$$
P_{s}(z)=Q+P^{(1)}(z)+P^{(2)}(z)+R(z)+V(z) .
$$

\section{Special Cases}

Case 1 (no reneging). Here we take $\gamma=0$. Then our model changes to a batch arrival single server vacation queue with server providing service in two fluctuating modes with breakdown and no reneging.
Then $k$ changes to $k=\lambda-\lambda C(z)$ and our equations in (46) reduce to

$$
\begin{aligned}
P^{(1)}(z)= & \frac{p_{1} Q\left[(\lambda-\lambda C(z)+\alpha)-\alpha B^{*}(\lambda-\lambda C(z))\right]}{\Delta} \\
& \times \frac{\left[1-S_{1}^{*}(\lambda-\lambda C(z)+\alpha)\right]}{\lambda-\lambda C(z)+\alpha} \\
P^{(2)}(z)= & \frac{p_{2} Q\left[(\lambda-\lambda C(z)+\alpha)-\alpha B^{*}(\lambda-\lambda C(z))\right]}{\Delta} \\
& \times \frac{\left[1-S_{2}^{*}(\lambda-\lambda C(z)+\alpha)\right]}{\lambda-\lambda C(z)+\alpha} \\
R(z)= & \frac{\alpha Q\left[p_{1} S_{1}^{*}(m)+p_{2} S_{2}^{*}(m)\right]}{\Delta} \\
& \times \frac{\left[\left\{1-\phi+\phi F^{*}(\lambda-\lambda C(z))\right\}-z\right]}{\Delta} \\
& \times\left[\frac{\left.1-B^{*}(\lambda-\lambda C(z))\right]}{\lambda-\lambda C(z)}\right] \\
& \times\left[\frac{\phi Q\left[m-\alpha B^{*}(\lambda-\lambda C(z))\right]\left[p_{1} S_{1}^{*}(m)+p_{2} S_{2}^{*}(m)\right]}{\lambda-\lambda C(z)}\right. \\
V(z)= & \frac{\left.F^{*}(\lambda-\lambda C(z))\right]}{\lambda-\lambda}
\end{aligned}
$$

where

$$
\begin{aligned}
\Delta= & -\left\{1-\phi+\phi F^{*}(\lambda-\lambda C(z))\right\}\left\{p_{1} S_{1}^{*}(m)+p_{2} S_{2}^{*}(m)\right\} \\
& -z \alpha B^{*}(\lambda-\lambda C(z)) \\
& \times\left[\frac{p_{1}\left(1-S_{1}^{*}(m)\right)+p_{2}\left(1-S_{2}^{*}(m)\right)}{m}\right]
\end{aligned}
$$

and $Q$ is given by

$$
\begin{aligned}
Q=( & {\left[p_{1} S_{1}^{*}(\alpha)+p_{2} S_{2}^{*}(\alpha)\right][1-\phi \lambda E(I) E(V)] } \\
& -[\alpha \lambda E(I) E(R)+\lambda E(I)] \\
& \left.\times\left[\frac{p_{1}\left(1-S_{1}^{*}(\alpha)\right)+p_{2}\left(1-S_{2}^{*}(\alpha)\right)}{\alpha}\right]\right) \\
& \times\left((1+\alpha E(R))\left\{p_{1} S_{1}^{*}(\alpha)+p_{2} S_{2}^{*}(\alpha)\right\}\right)^{-1} .
\end{aligned}
$$

Thus the stability condition $\rho$ is

$$
\begin{aligned}
\rho=( & \{\alpha \lambda E(I) E(R)+\lambda E(I)\} \\
& \times\left[\frac{p_{1}\left(1-S_{1}^{*}(\alpha)+p_{2}\left(1-S_{2}^{*}(\alpha)\right)\right)}{\alpha}\right] \\
& +\{\phi \lambda E(I) E(V)+\alpha E(R)\} \\
& \left.\times\left\{p_{1} S_{1}^{*}(\alpha)+p_{2} S_{2}^{*}(\alpha)\right\}\right) \\
& \times\left((1-\alpha E(R))\left\{p_{1} S_{1}^{*}(\alpha)+p_{2} S_{2}^{*}(\alpha)\right\}\right)^{-1}<1 .
\end{aligned}
$$


Case 2 (no breakdowns and no reneging). Here $\alpha=0, \gamma=$ 0 . Then the queuing model changes to a Batch arrival single server vacation queue with server providing service in two fluctuating modes.

Thus $m=\lambda-\lambda C(z), k=\lambda-\lambda C(z)$ and equations in (46) reduce to

$$
\begin{gathered}
P^{(1)}(z)=\frac{p_{1} Q\left[1-S_{1}^{*}(\lambda-\lambda C(z))\right]}{\Delta} \\
P^{(2)}(z)=\frac{p_{2} Q\left[1-S_{2}^{*}(\lambda-\lambda C(z))\right]}{\Delta} \\
V(z)=\left(\phi Q\left[p_{1} S_{1}^{*}(\lambda-\lambda C(z))+p_{2} S_{2}^{*}(\lambda-\lambda C(z))\right]\right. \\
\left.\times\left[1-F^{*}(\lambda-\lambda C(z))\right]\right) \times(\Delta)^{-1},
\end{gathered}
$$

where $\Delta=\left[1-\phi+\phi F^{*}(\lambda-\lambda C(z))\right]\left[p_{1} S_{1}^{*}(\lambda-\lambda C(z))+\right.$ $\left.p_{2} S_{2}^{*}(\lambda-\lambda C(z))\right]-z$.

The probability of idle time $Q$ is

$$
Q=1-\lambda E(I)\left[p_{1} E\left(G_{1}\right)+p_{2} E\left(G_{2}\right)+\phi E(V)\right] .
$$

Thus $\rho=\lambda E(I)\left[p_{1} E\left(G_{1}\right)+p_{2} E\left(G_{2}\right)+\phi E(V)\right]<1$, where $E\left(G_{1}\right)$ and $E\left(G_{2}\right)$ are the expected value of service completion in modes 1 and mode 2 , respectively.

Case 3 (no vacation). Here we take $\phi=0$. Consequently our probability generating functions for the server providing service in modes 1 and 2 at random point of time are given by

$$
\begin{aligned}
& P^{(1)}(z)=\frac{p_{1} Q\left[m-\alpha B^{*}(k)\right]}{\Delta}\left[\frac{1-S_{1}^{*}(m)}{m}\right] \\
& P^{(2)}(z)=\frac{p_{2} Q\left[m-\alpha B^{*}(k)\right]}{\Delta}\left[\frac{1-S_{2}^{*}(m)}{m}\right] .
\end{aligned}
$$

The steady state probability that the server is under repairs at random point of time is

$$
\begin{aligned}
R(z)= & \frac{\alpha Q\left[p_{1} S_{1}^{*}(m)+p_{2} S_{2}^{*}(m)\right](1-z)}{\Delta}\left[\frac{1-B^{*}(k)}{k}\right], \\
\Delta= & z-\left\{p_{1} S_{1}^{*}(m)+p_{2} S_{2}^{*}(m)\right\}-z \alpha B^{*}(m) \\
& \times\left[\frac{p_{1}\left(1-S_{1}^{*}(m)\right)+p_{2}\left(1-S_{2}^{*}(m)\right)}{m}\right] .
\end{aligned}
$$

Thus $Q$, the probability of idle time is

$$
\begin{aligned}
Q= & \left(\left\{p_{1} S_{1}^{*}(\alpha)+p_{2} S_{2}^{*}(\alpha)\right\}\right. \\
& -\{\alpha(\lambda E(I)-\gamma) E(R)+\lambda E(I)\} \\
& \left.\times\left[\frac{p_{1}\left(1-S_{1}^{*}(\alpha)\right)+p_{2}\left(1-S_{2}^{*}(\alpha)\right)}{\alpha}\right]\right) \\
& \times\left(\{1+\alpha E(R)\}\left\{p_{1} S_{1}^{*}(\alpha)+p_{2} S_{2}^{*}(\alpha)\right\}\right)^{-1}
\end{aligned}
$$

and the stability condition under which the steady state exists is

$$
\begin{aligned}
\rho=( & \{\alpha(\lambda E(I)-\gamma) E(R)+\lambda E(I)\} \\
& \times\left[\frac{p_{1}\left(1-S_{1}^{*}(\alpha)\right)+p_{2}\left(1-S_{2}^{*}(\alpha)\right)}{\alpha}\right] \\
& \left.+\alpha E(R)\left\{p_{1} S_{1}^{*}(\alpha)+p_{2} S_{2}^{*}(\alpha)\right\}\right) \\
& \times\left(\{1-\alpha E(R)\}\left\{p_{1} S_{1}^{*}(\alpha)+p_{2} S_{2}^{*}(\alpha)\right\}\right)^{-1} \\
< & 1 .
\end{aligned}
$$

Case 4 (server providing service in mode). In this case, we take $p_{1}=1, p_{2}=0$.

Thus our probability generating functions for server providing service in mode 1 , server under repairs, and server taking vacation are, respectively, given by (67), (68), and (69)

$$
\begin{gathered}
P^{(1)}(z)=\frac{\left[m-\alpha B^{*}(k)\right]}{\Delta}\left[\frac{1-S_{1}^{*}(m)}{m}\right], \\
R(z)=\frac{\alpha Q S_{1}^{*}(m)\left[\left\{1-\phi+\phi F^{*}(k)\right\}-z\right]}{\Delta}\left[\frac{1-B^{*}(k)}{k}\right], \\
V(z)=\frac{\phi Q\left[m-\alpha B^{*}(k)\right] S_{1}^{*}(m)}{\Delta}\left[\frac{1-F^{*}(k)}{k}\right]
\end{gathered}
$$

The probability of idle time $Q$ is

$$
\begin{aligned}
Q= & \left(S_{1}^{*}(\alpha)[1-\phi(\lambda E(I)-\gamma) E(V)]\right. \\
& \left.-[\alpha(\lambda E(I)-\gamma) E(R)+\lambda E(I)]\left[\frac{1-S_{1}^{*}(\alpha)}{\alpha}\right]\right) \\
& \times\left(\{1+\phi \gamma E(V)+\alpha E(R)\} S_{1}^{*}(\alpha)\right)^{-1} \\
\rho= & \left(\{\phi \lambda E(I) E(V)+\alpha E(R)\} S_{1}^{*}(\alpha)\right. \\
& \left.\quad+[\alpha(\lambda E(I)-\gamma) E(R)+\lambda E(I)]\left[\frac{1-S_{1}^{*}(\alpha)}{\alpha}\right]\right) \\
& \times\left(\{1+\phi \gamma E(V)+\alpha E(R)\} S_{1}^{*}(\alpha)\right)^{-1}<1 .
\end{aligned}
$$

The utilization factor of the system is given by

$$
\begin{aligned}
\rho= & \left(\{\phi \lambda E(I) E(V)+\alpha E(R)\} S_{1}^{*}(\alpha)\right. \\
& \left.+[\alpha(\lambda E(I)-\gamma) E(R)+\lambda E(I)]\left[\left(1-S_{1}^{*}(\alpha)\right) / \alpha\right]\right) \\
& \times\left(\{1+\phi \gamma E(V)+\alpha E(R)\} S_{1}^{*}(\alpha)\right)^{-1}<1 .
\end{aligned}
$$


Case 5 (no breakdown, no vacation and no reneging). The corresponding results for this particular case are obtained by putting $\alpha=0, \phi=0, \gamma=0$. Consequently $m=\lambda-\lambda C(z)$, $k=\lambda-\lambda C(z)$. Thus $S_{j}^{*}(0)=1, j=1,2, B^{*}(0)=1, F^{*}(0)=1$. With these substitutions in our main results, we obtain the following.

The probability generating functions of the queue size when the server is providing service in mode 1 and mode 2 are

$$
\begin{gathered}
P^{(1)}(z)=\frac{p_{1} Q\left[1-S_{1}^{*}(\lambda-\lambda C(z))\right]}{\Delta}, \\
P^{(2)}(z)=\frac{p_{2} Q\left[1-S_{2}^{*}(\lambda-\lambda C(z))\right]}{\Delta}, \\
\Delta=z-\left[p_{1} S_{1}^{*}(m)+p_{2} S_{2}^{*}(m)\right] .
\end{gathered}
$$

The idle time probability $Q$ is given by

$$
Q=1-\lambda E(I)\left\{p_{1} E\left(G_{1}\right)+p_{2} E\left(G_{2}\right)\right\} \text {. }
$$

Thus $\rho=\lambda E(I)\left\{p_{1} E\left(G_{1}\right)+p_{2} E\left(G_{2}\right)\right\}<1$ is the stability condition under which the steady state condition holds and $E\left(G_{j}\right)=\lim _{z \rightarrow 1}\left[\left(1-S_{j}^{*}(\lambda-\lambda C(z))\right) /(\lambda-\lambda C(z))\right] ; j=1,2$ is the expected value of service completion in mode 1 and mode 2 , respectively.

Case 6 (no breakdown, no vacation and no reneging and server providing service in single mode). The corresponding results for this particular case are obtained by putting $\alpha=0$, $\phi=0, \gamma=0$, and $p_{2}=0$. Consequently $p_{1}=1, m=\lambda-\lambda C(z)$, with these substitutions in our main results, we obtain the following.

The probability generating function of the queue size when the server is providing service in single mode

$$
\begin{aligned}
P^{(1)}(z) & =\frac{Q\left[1-S^{*}(\lambda-\lambda C(z))\right]}{\Delta}, \\
\Delta & =S^{*}(\lambda-\lambda C(z))-z .
\end{aligned}
$$

The idle time probability $Q$ is given by $Q=1-\lambda E(I) E(G)$ and $\rho=\lambda E(I) E(G)<1$, is the stability condition under which the steady state conditions are satisfied.

Equation (74) gives the probability generating function of the number of customers in the queue at random epoch for an $M^{X} / G / 1$ queueing system.

Case 7 (the service time, vacation completion time, and repair time are considered to follow exponential distribution). Here we take $S_{1}^{*}(m)=\mu_{1} /\left(\mu_{1}+m\right), S_{2}^{*}(m)=\mu_{2} /\left(\mu_{2}+m\right), B^{*}(k)=$ $\beta /(\beta+k), F^{*}(k)=\eta /(\eta+k)$, where $m=\lambda-\lambda C(z)+\alpha$, $k=\lambda-\lambda C(z)+\gamma-\gamma / z$.
The units of arrivals are also assumed to be one by one, such that $E(I)=1$. Also here $E(R)=1 / \beta$ and $E(V)=1 / \eta$. Thus our equations in (46) changes to

$$
\begin{aligned}
& P^{(1)}(z)=p_{1} Q\left[\frac{m(\beta+k)-\alpha \beta}{\beta+k}\right] \frac{1}{\mu_{1}+m} \\
& \times\left(z-\left\{\frac{(\eta+k)(1-\phi)+\phi \eta}{\eta+k}\right\}\right. \\
& \times\left\{\frac{p_{1} \mu_{1}}{\mu_{1}+m}+\frac{p_{2} \mu_{2}}{\mu_{2}+m}\right\} \\
& \left.-\frac{z \alpha \beta}{\beta+k}\left[\frac{p_{1}}{\mu_{1}+k}+\frac{p_{2}}{\mu_{2}+k}\right]\right)^{-1} \text {, } \\
& P^{(2)}(z)=p_{2} Q\left[\frac{m(\beta+k)-\alpha \beta}{\beta+k}\right] \frac{1}{\mu_{2}+m} \\
& \times\left(z-\left\{\frac{(\eta+k)(1-\phi)+\phi \eta}{\eta+k}\right\}\right. \\
& \times\left\{\frac{p_{1} \mu_{1}}{\mu_{1}+m}+\frac{p_{2} \mu_{2}}{\mu_{2}+m}\right\} \\
& \left.-\frac{z \alpha \beta}{\beta+k}\left[\frac{p_{1}}{\mu_{1}+k}+\frac{p_{2}}{\mu_{2}+k}\right]\right)^{-1} \text {, } \\
& R(z)=\alpha Q\left[\frac{p_{1} \mu_{1}}{\mu_{1}+m}+\frac{p_{2} \mu_{2}}{\mu_{2}+m}\right]\left[\frac{(1-\phi)+\phi \eta}{\eta+k}\right] \frac{1}{\beta+k} \\
& \times\left(z-\left\{\frac{(\eta+k)(1-\phi)+\phi \eta}{\eta+k}\right\}\right. \\
& \times\left\{\frac{p_{1} \mu_{1}}{\mu_{1}+m}+\frac{p_{2} \mu_{2}}{\mu_{2}+m}\right\} \\
& \left.-\frac{z \alpha \beta}{\beta+k}\left[\frac{p_{1}}{\mu_{1}+k}+\frac{p_{2}}{\mu_{2}+k}\right]\right)^{-1} \text {, } \\
& V(z)=\left(\phi Q\left[\frac{p_{1} \mu_{1}}{\mu_{1}+m}+\frac{p_{2} \mu_{2}}{\mu_{2}+m}\right]\left[\frac{m(\beta+k)-\alpha \beta}{\beta+k}\right]\right. \\
& \left.\times \frac{1}{\eta+k}\right) \times\left(z-\left\{\frac{(\eta+k)(1-\phi)+\phi \eta}{\eta+k}\right\}\right. \\
& \times\left\{\frac{p_{1} \mu_{1}}{\mu_{1}+m}+\frac{p_{2} \mu_{2}}{\mu_{2}+m}\right\} \\
& \left.-\frac{z \alpha \beta}{\beta+k}\left[\frac{p_{1}}{\mu_{1}+k}+\frac{p_{2}}{\mu_{2}+k}\right]\right)^{-1} \text {. }
\end{aligned}
$$

Probability that the server is providing service in mode 1 and mode 2 at a random point of time is

$$
\begin{aligned}
P^{(1)}(1)=Q & p_{1}\left[\lambda+\frac{\alpha(\lambda-\gamma)}{\beta}\right] \frac{1}{\mu_{1}+\alpha} \\
& \times\left(\left(\frac{p_{1} \mu_{1}}{\mu_{1}+\alpha}+\frac{p_{2} \mu_{2}}{\mu_{2}+\alpha}\right)\left[1-\frac{\phi(\lambda-\gamma)}{\eta}\right]\right. \\
& \left.-\left(\frac{\alpha(\lambda-\gamma)}{\beta}+\lambda\right)\left(\frac{p_{1}}{\mu_{1}+\alpha}\right)\left(\frac{p_{2}}{\mu_{2}+\alpha}\right)\right)^{-1},
\end{aligned}
$$




$$
\begin{aligned}
P^{(2)}(1)=Q p_{2} & {\left[\lambda+\frac{\alpha(\lambda-\gamma)}{\beta}\right] \frac{1}{\mu_{2}+\alpha} } \\
& \times\left(\left(\frac{p_{1} \mu_{1}}{\mu_{1}+\alpha}+\frac{p_{2} \mu_{2}}{\mu_{2}+\alpha}\right)\left[1-\frac{\phi(\lambda-\gamma)}{\eta}\right]\right. \\
& \left.-\left(\frac{\alpha(\lambda-\gamma)}{\beta}+\lambda\right)\left(\frac{p_{1}}{\mu_{1}+\alpha}\right)\left(\frac{p_{2}}{\mu_{2}+\alpha}\right)\right)^{-1} .
\end{aligned}
$$

The probability that the server is under repairs at random point of time is

$$
\begin{aligned}
R(1)= & \left(\frac{p_{1} \mu_{1}}{\mu_{1}+\alpha}+\frac{p_{2} \mu_{2}}{\mu_{2}+\alpha}\right)\left[1-\frac{(\lambda-\gamma) \phi}{\eta}\right] \frac{1}{\beta} \\
& \times\left(\left(\frac{p_{1} \mu_{1}}{\mu_{1}+\alpha}+\frac{p_{2} \mu_{2}}{\mu_{2}+\alpha}\right)\left[1-\frac{\phi(\lambda-\gamma)}{\eta}\right]\right. \\
& \left.\quad-\left(\frac{\alpha(\lambda-\gamma)}{\beta}+\lambda\right)\left(\frac{p_{1}}{\mu_{1}+\alpha}+\frac{p_{2}}{\mu_{2}+\alpha}\right)\right)^{-1} .
\end{aligned}
$$

Probability that the server is on vacation at random point of time is

$$
\begin{aligned}
V(1)= & \phi Q\left(\frac{p_{1} \mu_{1}}{\mu_{1}+\alpha}+\frac{p_{2} \mu_{2}}{\mu_{2}+\alpha}\right)\left[\lambda+\frac{\alpha(\lambda-\gamma) \phi}{\beta}\right] \frac{1}{\eta} \\
\times & \left(\left(\frac{p_{1} \mu_{1}}{\mu_{1}+\alpha}+\frac{p_{2} \mu_{2}}{\mu_{2}+\alpha}\right)\left[1-\frac{\phi(\lambda-\gamma)}{\eta}\right]\right. \\
& \left.-\left(\frac{\alpha(\lambda-\gamma)}{\beta}+\lambda\right)\left(\frac{p_{1}}{\mu_{1}+\alpha}+\frac{p_{2}}{\mu_{2}+\alpha}\right)\right)^{-1} .
\end{aligned}
$$

The probability that the server is idle but available in the system is given by

$$
\begin{aligned}
Q= & \left(\left[\frac{p_{1} \mu_{1}}{\mu_{1}+\alpha}+\frac{p_{2} \mu_{2}}{\mu_{2}+\alpha}\right]\left[1-\frac{\phi(\lambda-\gamma)}{\eta}\right]\right. \\
& \left.-\left[\frac{\alpha(\lambda-\gamma)}{\beta}+\lambda\right]\left[\frac{p_{1}}{\mu_{1}+\alpha}+\frac{p_{2}}{\mu_{2}+\alpha}\right]\right) \\
& \times\left(\left(1+\frac{\phi \gamma}{\eta}+\frac{\alpha}{\beta}\right)\left(\frac{p_{1} \mu_{1}}{\mu_{1}+\alpha}+\frac{p_{2} \mu_{2}}{\mu_{2}+\alpha}\right)\right)^{-1}, \\
\rho= & \left(\left[\frac{p_{1} \mu_{1}}{\mu_{1}+\alpha}+\frac{p_{2} \mu_{2}}{\mu_{2}+\alpha}\right]\left[\frac{\phi \lambda}{\eta}+\frac{\alpha}{\beta}\right]\right. \\
& \left.+\left[\frac{\alpha(\lambda-\gamma)}{\beta}+\lambda\right]\left[\frac{p_{1}}{\mu_{1}+\alpha}+\frac{p_{2}}{\mu_{2}+\alpha}\right]\right) \\
& \times\left(\left(1+\frac{\phi \gamma}{\eta}+\frac{\alpha}{\beta}\right)\left(\frac{p_{1} \mu_{1}}{\mu_{1}+\alpha}+\frac{p_{2} \mu_{2}}{\mu_{2}+\alpha}\right)\right)^{-1}<1 .
\end{aligned}
$$

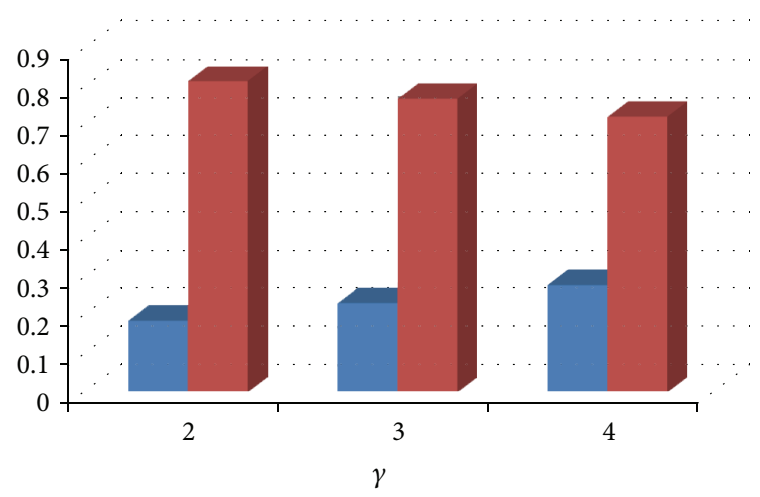

- $Q$

- $\rho$

FIGURE 1: Effect of reneging parameter $\gamma$ on the proportion of idle time $Q$ and utilization factor $\rho$ (when $\alpha=2, \beta=6, \eta=5, \phi=0.5$ ).

And the utilization factor is

$$
\begin{aligned}
Q= & \left(\left[\frac{p_{1} \mu_{1}}{\mu_{1}+\alpha}+\frac{p_{2} \mu_{2}}{\mu_{2}+\alpha}\right]\left[1-\frac{\phi(\lambda-\gamma)}{\eta}\right]\right. \\
& \left.-\left[\frac{\alpha(\lambda-\gamma)}{\beta}+\lambda\right]\left[\frac{p_{1}}{\mu_{1}+\alpha}+\frac{p_{2}}{\mu_{2}+\alpha}\right]\right) \\
& \times\left(\left(1+\frac{\phi \gamma}{\eta}+\frac{\alpha}{\beta}\right)\left(\frac{p_{1} \mu_{1}}{\mu_{1}+\alpha}+\frac{p_{2} \mu_{2}}{\mu_{2}+\alpha}\right)\right)^{-1}<1 .
\end{aligned}
$$

\section{Numerical Example}

We illustrate a numerical example in order to see the effect and validity of our results of the different parameters used in our model, namely, the failure rate $\alpha$, reneging rate $\gamma$, the completion of repairs parameter $\beta$, and completion of vacation $\eta$ on the utilization factor $\rho$ and on the probabilities of various states of the system, namely, the working state, failure state, vacation, and idle state. We choose all the values of the parameters arbitrarily such that the stability condition (54) is not violated.

We consider the special Case 6 for our numerical illustration. We fix the values of arrival rate $\lambda$, service rates $\mu_{1}$ and $\mu_{2}$, the probabilities of providing service in modes 1 and $2 p_{1}$ and $p_{2}$, and probability of server taking vacation $\phi$.

Numerical Table 1 shows that for fixed $\beta$ (repair completion rate) and fixed $\alpha$ (breakdown rate), the effect of increasing the reneging rate $(\gamma)$ and decrease in the vacation completion rate $(\eta)$ shows a decrease in the utilization factor $(\rho)$ and an increase in the idle time $Q$. It also shows that due to these changes, there is decrease in the probability of the server providing service in modes 1 and 2 , respectively. It is clear from Figure 1 that as the rate of reneging increases, the proportion of server busy period $(\rho)$ also increases while 
TABLE 1: $\lambda=4, \mu_{1}=8, \mu_{2}=10, p_{1}=0.4, p_{2}=0.6$, and $\phi=0.5$.

\begin{tabular}{llllllllll}
\hline & $\beta$ & $\gamma$ & $\eta$ & $\rho$ & $Q$ & $P^{(1)}(1)$ & $P^{(2)}(1)$ & $R(1)$ & 0.1739 \\
\hline \multirow{2}{*}{$\alpha=2$} & 6 & 2 & 5 & 0.8123 & 0.1877 & 0.1485 & 0.1856 & 0.3043 \\
& 6 & 3 & 4 & 0.7662 & 0.2338 & 0.1237 & 0.1546 & 0.1708 & 0.3171 \\
& 6 & 4 & 3 & 0.7195 & 0.2805 & 0.0976 & 0.1220 & 0.1667 & 0.3333 \\
\hline \multirow{2}{*}{$\alpha=3$} & 6 & 2 & 5 & 0.8510 & 0.1490 & 0.1426 & 0.1809 & 0.2353 & 0.2941 \\
& 6 & 3 & 4 & 0.7957 & 0.2043 & 0.1164 & 0.1477 & 0.2333 & 0.2999 \\
& 6 & 4 & 3 & 0.7404 & 0.2596 & 0.0895 & 0.1136 & 0.2307 & 0.3076 \\
\hline \multirow{2}{*}{$\alpha=4$} & 6 & 2 & 5 & 0.8837 & 0.1163 & 0.1370 & 0.1762 & 0.2859 & 0.2859 \\
& 6 & 3 & 4 & 0.8208 & 0.1792 & 0.1096 & 0.1644 & 0.2858 & 0.2858 \\
& 6 & 4 & 3 & 0.7589 & 0.2411 & 0.0822 & 0.1057 & 0.2856 & 0.2856 \\
\hline
\end{tabular}

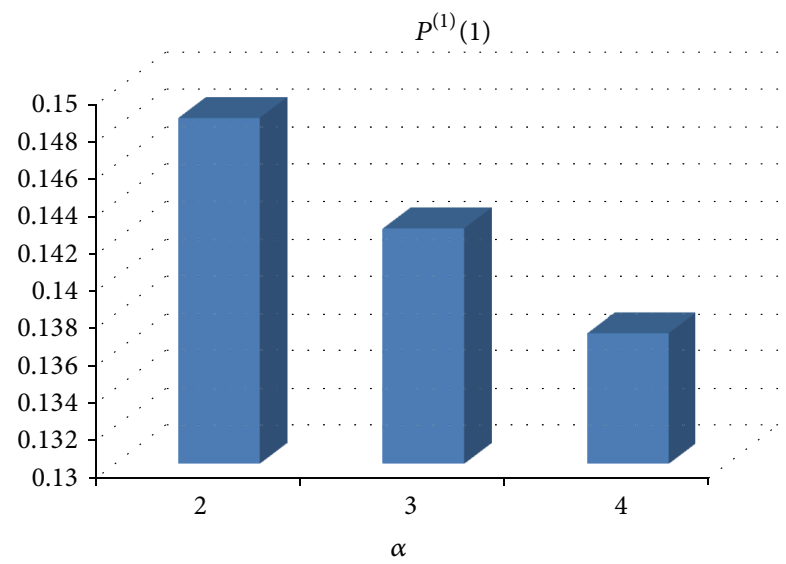

FIGURE 2: Effect of breakdown parameter $\alpha$ on the probability that the server is providing service in mode 1 (when $\beta=6, \gamma=2, \eta=5$ ).

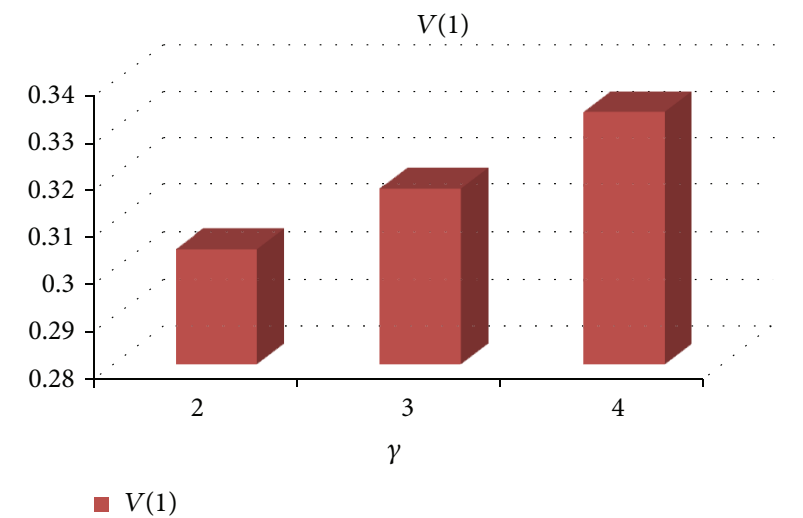

Figure 4: Effect of $\gamma$ and $\eta$ on the probability that server is on vacation $(\alpha=2, \beta=6, \eta=2,3,4)$.

\section{Conflict of Interests}

The authors declare that there is no conflict of interests regarding the publication of this paper.

\section{References}

[1] Y. Levy and U. Yechiali, "An M/M/s queue with server vacations," INFOR Journal, vol. 14, no. 2, pp. 153-163, 1976.

[2] H. Takagi, "Time-dependent analysis of $M / G / 1$ vacation models with exhaustive service," Queueing Systems, vol. 6, no. 1, pp. 369389, 1990.

[3] B. T. Doshi, "Queueing systems with vacations-a survey," Queueing Systems, vol. 1, no. 1, pp. 29-66, 1986.

[4] K. C. Madan, "On a $M^{X} / M^{b} / 1$ queuing system with general vacation times," International Journal of Information and Management Sciences, vol. 2, no. 1, pp. 51-60, 1991.

[5] K. C. Madan, "On a single server queue with two -stage heterogeneous service and binomial schedule server vacations," The Egyptian Statistical Journal, vol. 44, no. 1, pp. 39-55, 2000.

[6] K. C. Madan, Z. R. Al-Rawi, and A. D. Al-Nasser, "On M ${ }^{X} /(G 2, G 1) / 1 / G(B S) / V_{S}$ vacation queue with two types of general heterogeneous service," Journal of Applied Mathematics and Decision Sciences, vol. 2005, no. 3, pp. 123-135, 2005. 
[7] D. P. Gaver, "A waiting line with interrupted service including priorities," Journal of the Royal Statistical Society B, vol. 24, pp. 73-90, 1962.

[8] A. Aissani and J. R. Artalejo, "On the single server retrial queue subject to breakdowns," Queueing Systems, vol. 30, no. 3-4, pp. 309-321, 1998.

[9] J. Wang, B. Liu, and J. Li, "Transient analysis of an $M / G / 1$ retrial queue subject to disasters and server failures," European Journal of Operational Research, vol. 189, no. 3, pp. 1118-1132, 2008.

[10] J. C. Ke, "Batch arrival queues under vacation policies with server breakdowns and startup/closedown times," Applied Mathematical Modelling, vol. 31, no. 7, pp. 1282-1292, 2007.

[11] Y. Tang, "A single-server $M / G / 1$ queueing system subject to breakdowns-some reliability and queueing problems," Microelectronics Reliability, vol. 37, no. 2, pp. 315-321, 1997.

[12] W. J. Gray, P. Wang, and M. Scott, "A queuing model with multiple types of server breakdowns," Quality Technology of Quantitative Management, vol. 1, no. 2, pp. 245-255, 2004.

[13] R. F. Khalaf, K. C. Madan, and C. A. Lukas, "An $M^{X} / G / 1$ with Bernoulli schedule general vacation times, general extended vacations, random breakdown, general delay time for repairs to start and general repair times," Journal of Mathematical Research, vol. 3, no. 4, pp. 8-20, 2011.

[14] D. J. Daley, "General customer impatience in the queue $G I / G / 1$," Journal of Applied Probability, vol. 2, no. 1, pp. 186-205, 1965.

[15] E. Altman and U. Yechiali, "Analysis of customers' impatience in queues with server vacations," Queueing Systems, vol. 52, no. 4, pp. 261-279, 2006.

[16] J. Bae, S. Kim, and E. Y. Lee, "The virtual waiting time of the $M / G / 1$ queue with impatient customers," Queueing Systems, vol. 38, no. 4, pp. 485-494, 2001.

[17] C. J. Ancker Jr. and A. V. Gafarian, "Some queuing problems with balking and reneging," Operation Research, vol. 11, no. 1, pp. 88-100, 1962.

[18] A. Choudhury and P. Medhi, "Balking and reneging in multiserver Markovian queuing systems," International Journal of Mathematics in Operational Research, vol. 3, no. 4, pp. 377-394, 2011.

[19] Y. Zhang, D. Yue, and W. Yue, "Analysis of an $M / M / 1 / N$ queue with balking, reneging and server vacations," in Proceedings of the 5th International Symposium on OR and its Applications, pp. 37-47, 2005. 


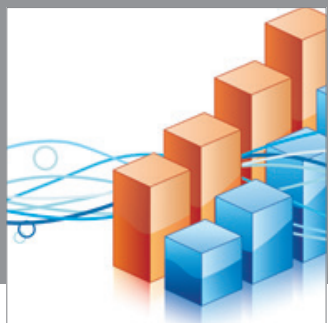

Advances in

Operations Research

mansans

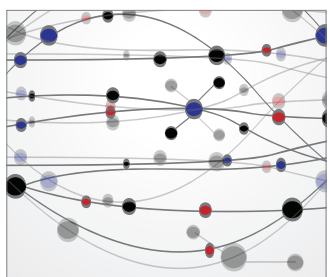

The Scientific World Journal
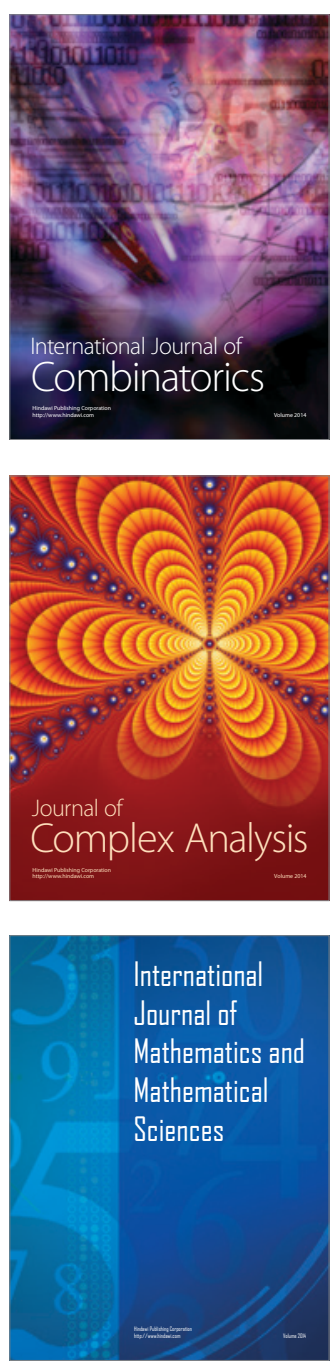
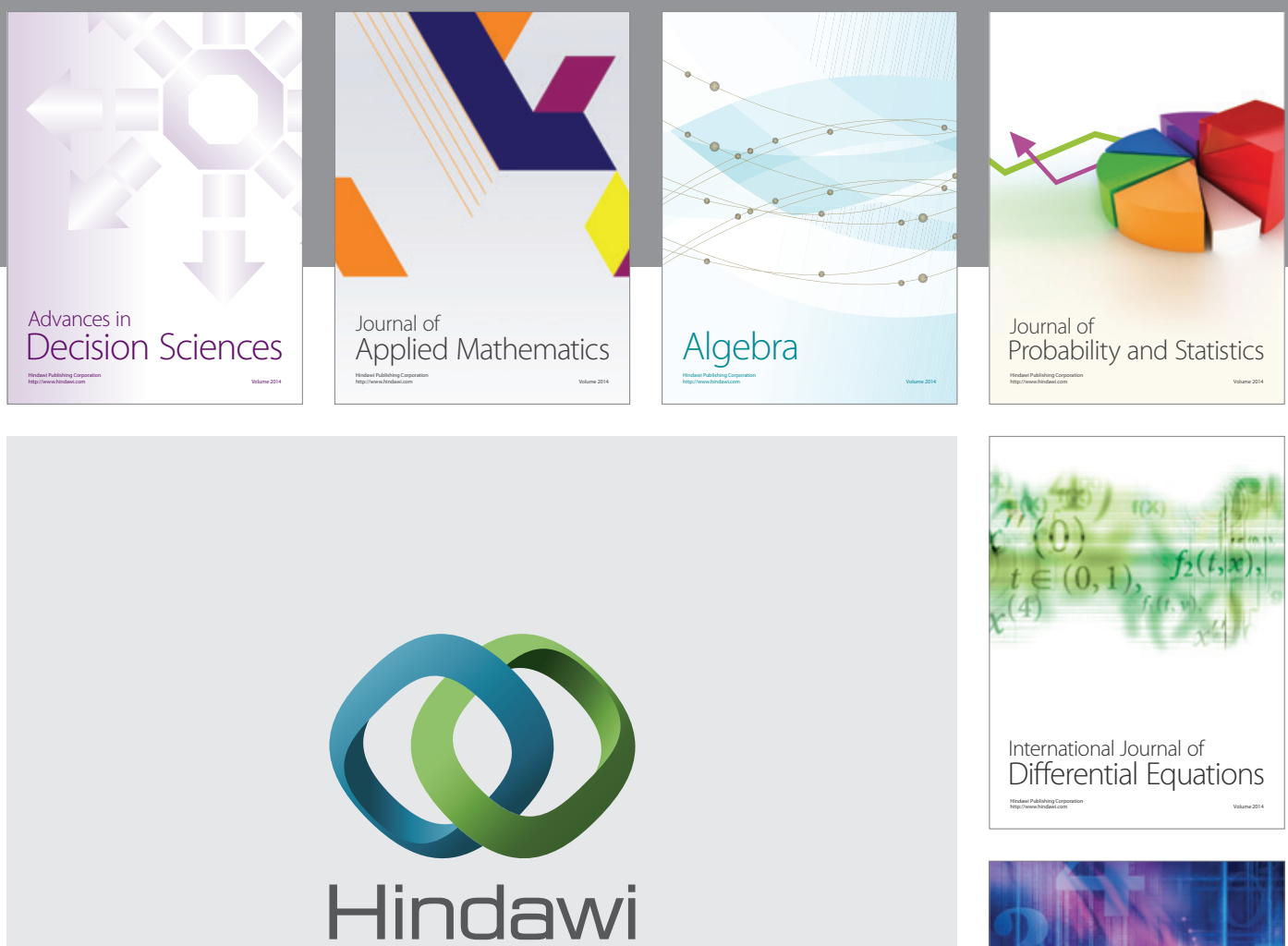

Submit your manuscripts at http://www.hindawi.com
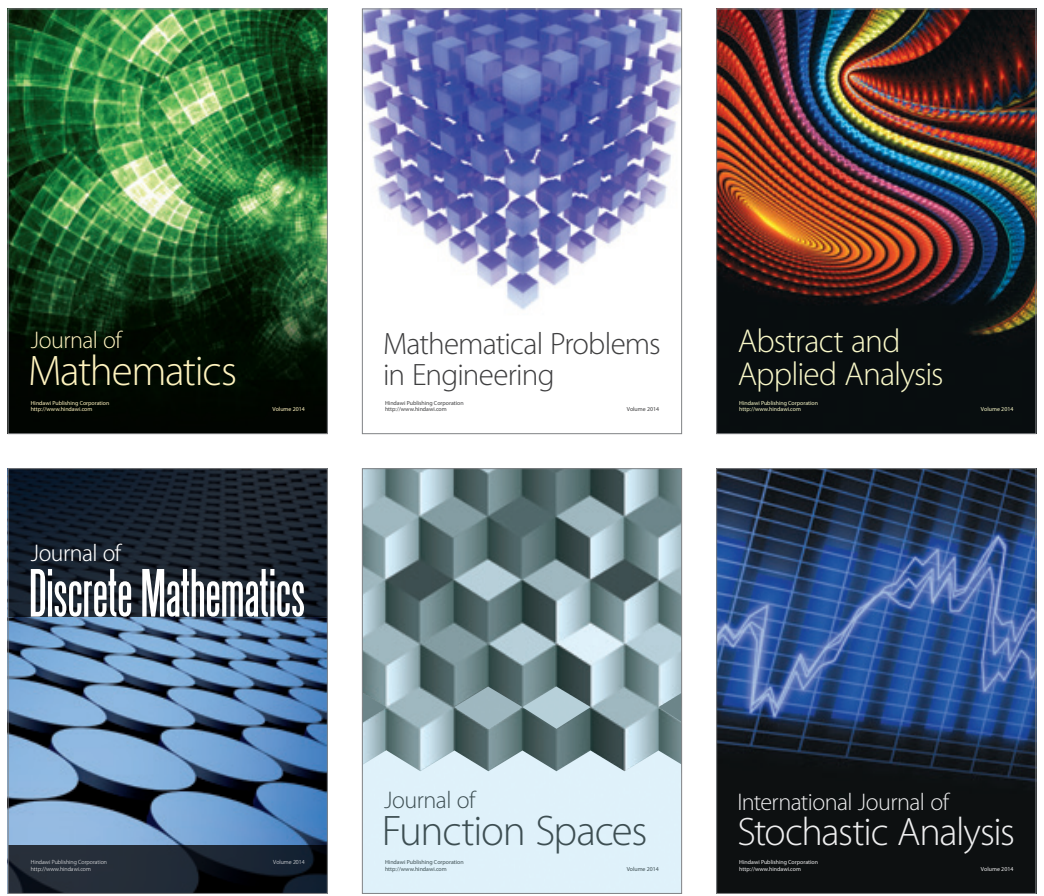

Journal of

Function Spaces

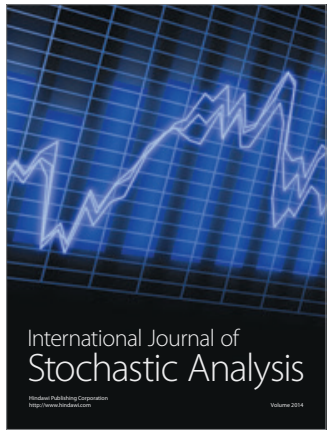

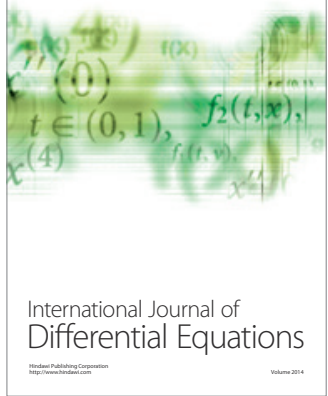
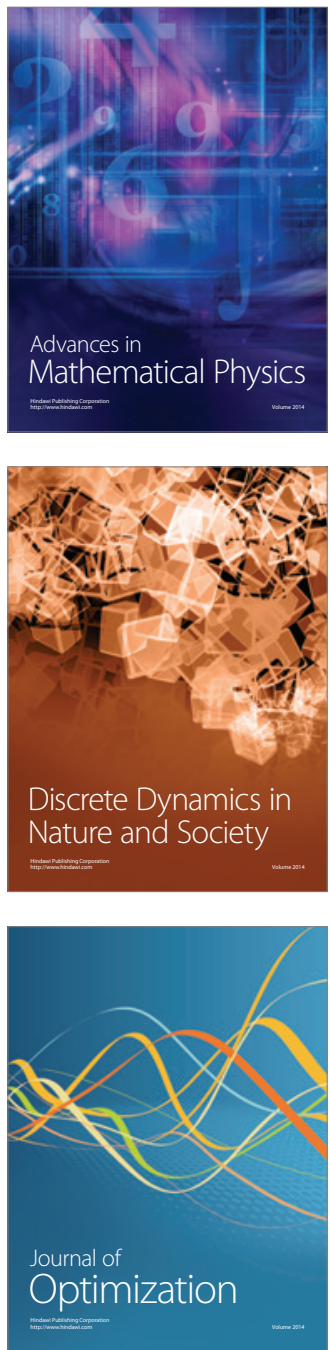